Lepr Rev (1990) 61, 171-179

\title{
Leprosy deformities: experience in Molai Leprosy Hospital, Maiduguri, Nigeria
}

\author{
B B IYERE \\ Molai Leprosy Hospital, PMB 1332 Molai, Maiduguri, Borno \\ State, Nigeria
}

Accepted for publication 2 October 1989

\begin{abstract}
Summary A total of 410 patients ( 288 males, 122 females) aged between 9 and 60 years with an average age of 32.5 years were assessed for deformities of the eyes, hands and feet. The objectives were to find out the number and types of leprosy deformities in the leprosy population of the hospital, the proportion of those deformed among them and to establish the deformity baseline for the hospital. The study lasted 1 year, $38.78 \%$ (26.59\% males, $12.20 \%$ females) of those investigated had one or more deformities. Apart from plantar and palmar insensitivity which accounted for $17.91 \%$ and $17 \cdot 24 \%$ of all deformities, the most frequent deformities were mobile claw hand $12.94 \%$, plantar ulcers $10 \cdot 78 \%$ and palmar ulcers $5.97 \%$ respectively. With the exception of eye deformities, males accounted for a higher proportion of all deformities. Hand deformities were the most frequent of the three parts of the body studied. The patients' problems were highlighted and the need for adequate management and self-care were emphasized.
\end{abstract}

\section{Introduction}

Leprosy is a chronic infectious disease almost restricted to man. It is caused by Mycobacterium leprae and it commonly affects the peripheral nerves and the skin. The estimated total number of leprosy patients in the world is about $15,000,000 .^{1}$

Deformity is an alteration in the form, shape or appearance of the affected part of the body. ${ }^{2}$ Leprosy is perhaps better known to the public because of the mutilation and crippling deformities it causes. Many people view these mutilations and gross deformities, which are the result of neglected or poorly managed leprosy, as an integral part of the disease process. This perhaps explains the enormous fear and stigma that the disease instills into members of an ill-informed public.

Disabilities and deformity in leprosy constitute a major problem in the management of the disease, ${ }^{3}$ yet they are not an inevitable result of the disease except in a few cases of untreated, advanced lepromatous leprosy. ${ }^{4}$

About $25 \%$ of leprosy patients who are not treated at an early stage of the disease 
develop deformities of the hands and feet. And these deformities disfigure, stigmatize and disrupt the lives of the patients and could constitute a handicap for a person's whole life. ${ }^{1}$

Leprosy patients with deformities are a common sight in our public places such as supermarkets, roundabouts, squares, churches and mosques. They patronize these places to display their plight to the public and to solicit alms and solace and perhaps to seek an understanding.

Molai Leprosy Hospital is on the outskirts of Maiduguri, Borno State capital, in Nigeria. It is the only such hospital in the entire state. Patients are drawn from the entire state and from neighbouring states.

This study was motivated by the presence of the many leprosy patients with deformities. The objectives of the study were to find out the number and types of deformities in our leprosy patients, the proportion of those with deformities among the entire patients and, above all, to establish a deformity baseline data for the hospital, upon which the disability prevention programme could be improved and assessed from time to time.

\section{Materials and methods}

Leprosy patients who reported to our hospital between 1 July 1987 and 30 June 1988 were used for the study. All the patients who attended the diagnostic skin clinic of the hospital and were confirmed as having leprosy were referred to the physiotherapy department for physical examination for leprosy deformities and assessment of deformities. All the patients were reviewed at 4-month intervals except for outpatients who had some complaints, or those admitted into the wards for one reason or another. They were reviewed as the patients, condition dictated.

Every patient had a physical examination and assessment which entailed examination of the face, hands and feet. The eye examination involved tests for corneal sensation and closure.

The hands were examined for palmar sensation, absorption of digits, finger/thumb weakness, contractures, palmar ulcers, including injury, and deep cracks. The hands were also examined and assessed for wrist drop, mobile claw fingers and thumb.

The feet were examined and information recorded about plantar sensation, foot weakness, claw toes, plantar ulcer, absorption of digits, foot drop and contractures.

\section{Assumptions for the purpose of this study}

Incomplete eye closure was one in which the patient was unable to close his/her eyes in such a way that the eyelids did not meet. Eyelid weakness was one in which the patient was able to close the eyes without any lid gap but was unable to do so when the eyelids were gently pulled apart.

Contracture was regarded as stiff joint ${ }^{4}$ and no reasonable range of movement was possible either actively or passively through such joints. Mobile claw fingers and thumb were one that were held in hyperextension of the metacarpophalangeal ( $\mathrm{mcp}$ ) joint and flexion of the proximal interphalangeal (PIP) joints and distal interphalangeal (DIP) 
joints 5 and the joints of such fingers could completely or almost completely be straightened..$^{4,6}$

Ulcer included injury and deep open cracks large enough to be scored grade two in the World Health Organization (WHO) disability grading system as quoted in one study. ${ }^{4}$

A mobile wrist drop was one that could be moved passively through its full range without or with very minimal resistance.

Foot drop was one in which the foot seemed 'too long' in such a way that when the patient lifted the foot up in walking, the toes trailed on the ground ${ }^{7}$ and the patient was unable to pull the foot up. ${ }^{8}$ A mobile foot drop was one that was able to be moved through its full range passively without or with very little resistance.

Finger, thumb, wrist and foot weakness were regarded as those in which a full range of movements were possible actively but that could not resist reasonable resistance.

\section{Sensory tests}

FACE-EYE

The whole procedure was explained to the patient, and after the patient showed understanding of the procedure and after two trials, the actual tests were conducted.

The patient's face was supported with one hand and the patient was asked to look up to the opposite side being tested. A piece of tissue paper was then used to gently touch the limbus of the cornea, approaching from the side, care was taken not to touch the eye lashes. If the patient blinked, it was recorded present, if no blink, corneal sensation was recorded absent. ${ }^{4,8}$ Incomplete eye closure (lagophthalmos) was tested by asking the patient to close his/her eyes as tightly as possible. If the patient closed the eyes in such a way that there was a lid gap, it was recorded as lagophthalmos. ${ }^{6}$

For eye weakness, the patient was asked to close his/her eyes as tightly as possible while the investigator tried to pull the eye lids gently apart. If the patient was unable to resist the pull, it was recorded weak. All tests were carried out while the patients were sitting.

\section{THE HAND}

The back of the patient's fingers and thumb being tested was supported with the palm of the investigator's left hand. Care was taken to ensure that there were no joint movements. The procedure was explained and demonstrated with the patient's eyes open. After the patient had shown understanding of the procedure, his/her face was covered with an opaque file jacket so that the patient did not see what was being done. A ballpoint pen held upright was used to gently dent different places of the palm, fingers and thumb and the patient was asked to touch the dented place with the tip of one finger indicating that he/ she felt the touch. ${ }^{6,8}$

Finger weakness was tested by placing a card (hospital) between the little and ring fingers of the patient's hand. The patient was asked to hold it as tightly as possible while the card was gently pulled out. If the patient was unable to resist the pull, the finger was recorded as weak. For the thumb, the patient was asked to move the thumb up in such a way that the base of the thumb was fully across and out while the thumb was straight. Pressure was then applied at the side of the thumb in this position. If unable to resist the pressure, it was recorded as weak. Weakness of the wrist was determined by asking the 
patient to pull the wrist back fully while gentle pressure was applied downwards. If he/she was unable to resist, it was regarded as weak. ${ }^{8}$

\section{THE FOOT}

Plantar sensation was tested for by indenting the sole of the foot in different places with a ballpoint pen held upright. The foot being examined was bent at the knee with the lateral border of the foot resting on the knee of the other leg. With the patient's face covered with an opaque file jacket, and after the procedure had been explained and the patient showed an understanding, the tip of the ballpoint pen was used to gently dent the sole at different places. The patient was asked to touch the place with the tip of one finger any time he/she felt the denting. ${ }^{6,8}$

Foot weakness was determined by asking the patient to pull up the foot fully while gentle pressure was applied at the top of foot. ${ }^{8}$ If the patient was unable to resist the pressure and yet when the patient walked, the toes did not trail on the ground, it was recorded as weak. All the data were recorded on the individual patient's sheet and were updated anytime the patient was reviewed. The final data was compiled at the end of the study.

For the purpose of this study, where a patient had a deformity and if eventually it degenerated to something worse, i.e. weakness of the little finger without discernible clawing to mobile clawing, it was the mobile clawing that was recorded against the patient in the final data. Equally for the purpose of this investigation, where a patient had the same deformity bilaterally, i.e. plantar ulcer, it was simply recorded as a plantar ulcer. These assumptions were largely informed by the fact that the study was about to find out what deformities patients presented with, during the period of investigation.

The lower and upper age limits of the patients were determined by the age limits of patients studied. Also 1 July 1987 to 30 June 1988 were chosen so that the duration of the study lasted 1 year.

\section{Results}

The 410 leprosy cases ( 288 or $70.24 \%$ males, 122 or $29 \cdot 76 \%$ females) were seen in the hospital between 1 July 1987 and 30 June 1988. Their ages ranged between 9 and 60 years with an average of 32.5 . They were made up of both new and old patients who either reported to the hospital on their own volition or were referred from other clinics in Borno

Table 1. Percentage of patients with deformities amongst 410 leprosy patients

\begin{tabular}{|c|c|c|c|c|c|c|}
\hline \multirow[b]{2}{*}{ Deformity/No deformity } & \multicolumn{2}{|c|}{ Male } & \multicolumn{2}{|c|}{ Female } & \multicolumn{2}{|c|}{ Total } \\
\hline & No. & $\%$ & No. & $\%$ & No. & $\%$ \\
\hline Deformities absent & 179 & 43.66 & 72 & $17 \cdot 56$ & 251 & $61 \cdot 22$ \\
\hline Deformities present & 109 & $26 \cdot 59$ & 50 & $12 \cdot 20$ & 159 & $38 \cdot 78$ \\
\hline Total leprosy cases assessed & 288 & $70 \cdot 25$ & 122 & $29 \cdot 76$ & 410 & 100 \\
\hline
\end{tabular}


State or from neighbouring states due to complications or for the confirmation of a diagnosis.

Table 1 and Figure 1 show the number and percentage of patients with deformities among the entire 410 leprosy population of the hospital; $159(38.78 \%)$ of the total number studied were found to have one or more deformities, $251(61 \cdot 22 \%)$ of the total patients were free of deformities.

Table 2 displays the various deformities found in the 159 patients with deformities, and indicates that the four most frequent deformities were plantar insensitivity (108 or

Table 2. Types of deformities found in 159 leprosy patients with one or more deformities

\begin{tabular}{|c|c|c|c|c|c|c|}
\hline \multirow[b]{2}{*}{ Types of deformities } & \multicolumn{2}{|c|}{ Male } & \multicolumn{2}{|c|}{ Female } & \multicolumn{2}{|c|}{ Total } \\
\hline & No. & $\%$ & No. & $\%$ & No. & $\%$ \\
\hline \multicolumn{7}{|l|}{ FACE } \\
\hline Eyelid weakness & 11 & $2 \cdot 7$ & 34 & $17 \cdot 6$ & 45 & $7 \cdot 46$ \\
\hline Corneal insensitivity & 7 & $1 \cdot 7$ & 1 & $0 \cdot 5$ & 8 & $1 \cdot 33$ \\
\hline Lagophthalmos & 9 & $2 \cdot 2$ & 6 & $3 \cdot 1$ & 15 & $2 \cdot 49$ \\
\hline \multicolumn{7}{|l|}{ HAND } \\
\hline Palmar insensitivity & 73 & $17 \cdot 8$ & 31 & $16 \cdot 1$ & 104 & $17 \cdot 24$ \\
\hline Absorption & 13 & $3 \cdot 2$ & 7 & $3 \cdot 6$ & 20 & $3 \cdot 32$ \\
\hline Contracture & 18 & $4 \cdot 4$ & 4 & $2 \cdot 1$ & 22 & 3.65 \\
\hline Palmar ulcer & 21 & $5 \cdot 1$ & 15 & $7 \cdot 8$ & 36 & 5.97 \\
\hline Wrist drop & 6 & $1 \cdot 5$ & 2 & $1 \cdot 0$ & 8 & $1 \cdot 33$ \\
\hline Mobile claw hand & 58 & $14 \cdot 1$ & 20 & $10 \cdot 4$ & 78 & $12 \cdot 94$ \\
\hline Weakness & 17 & $4 \cdot 1$ & 12 & $6 \cdot 2$ & 29 & $4 \cdot 81$ \\
\hline \multicolumn{7}{|l|}{ FOOT } \\
\hline Plantar insensitivity & 76 & $18 \cdot 5$ & 32 & $16 \cdot 6$ & 108 & $17 \cdot 91$ \\
\hline Mobile claw toes & 5 & $1 \cdot 2$ & 0 & $0 \cdot 0$ & 5 & 0.83 \\
\hline Plantar ulcer & 50 & $12 \cdot 2$ & 15 & $7 \cdot 8$ & 65 & $10 \cdot 78$ \\
\hline Absorption & 13 & $3 \cdot 2$ & 4 & $2 \cdot 1$ & 17 & $2 \cdot 82$ \\
\hline Foot drop & 24 & 5.9 & 7 & 3.6 & 31 & $5 \cdot 14$ \\
\hline Contracture & 3 & $0 \cdot 7$ & 2 & $1 \cdot 0$ & 5 & $0 \cdot 83$ \\
\hline Weakness & 6 & $1 \cdot 5$ & 1 & $0 \cdot 5$ & 7 & $1 \cdot 16$ \\
\hline Total & 410 & 100 & 193 & 100 & 603 & 100 \\
\hline
\end{tabular}

Table 3. Summary of 603 deformities in face, hands and feet amongst 159 leprosy patients

\begin{tabular}{|c|c|c|c|c|c|c|}
\hline \multirow[b]{2}{*}{ Types of deformity } & \multicolumn{2}{|c|}{ Male } & \multicolumn{2}{|c|}{ Female } & \multicolumn{2}{|c|}{ Total } \\
\hline & No. & $\%$ & No. & $\%$ & No. & $\%$ \\
\hline Face & 27 & $6 \cdot 6$ & 41 & $21 \cdot 20$ & 68 & $11 \cdot 28$ \\
\hline Hand & 206 & $50 \cdot 2$ & 91 & $47 \cdot 20$ & 297 & $49 \cdot 25$ \\
\hline Foot & 177 & $43 \cdot 20$ & 61 & $31 \cdot 60$ & 238 & 39.47 \\
\hline Total & 410 & 100 & 193 & 100 & 603 & 100 \\
\hline
\end{tabular}




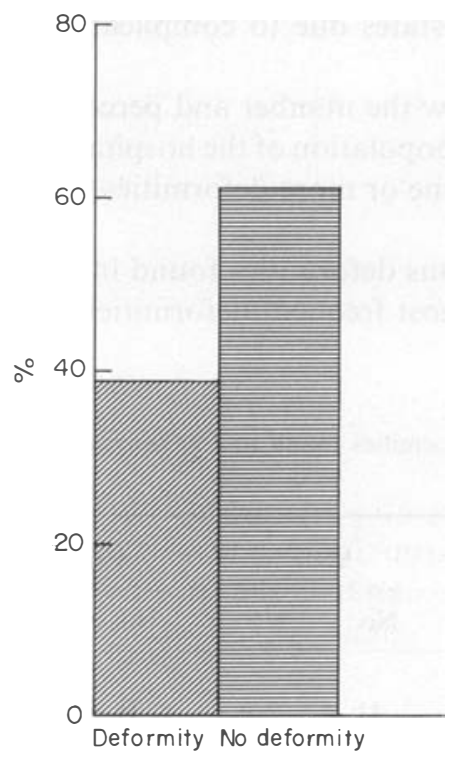

Figure 1. Percentage of patients with deformities amongst 410 leprosy patients.

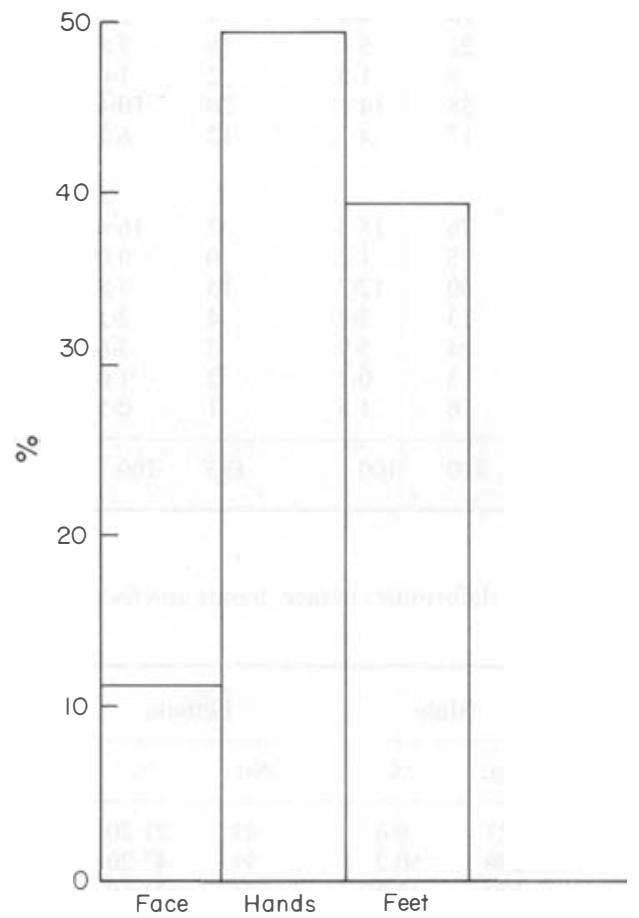

Figure 2. Percentage of deformities found in face, hands and feet amongst 159 patients with a total of 603 deformities. 
$17 \cdot 91 \%$ ), palmar insensitivity (104 or $17 \cdot 24 \%$ ), mobile claw hand (78 or $12.94 \%$ ) and plantar ulcer ( 65 or $10.78 \%$ ), of all deformities.

Table 2 also indicates that the two most common deformities of the face, hands and feet were eye weakness $45(7 \cdot 46 \%)$ and lagophthalmos $15(2 \cdot 49 \%)$; palmar insensitivity $104(17 \cdot 23 \%)$ and mobile claw hand $78(12.94 \%)$; plantar insensitivity $108(17 \cdot 91 \%)$ and plantar ulcer $65(10.78 \%)$ of all deformities.

Table 3 and Figure 2 show the total deformities found in face, hands and feet. Most patients presented with hand deformities, $297(49 \cdot 25 \%) ; 238(39 \cdot 47 \%)$ were foot deformities, while face deformities accounted for $68(11 \cdot 28 \%)$.

Figure 3 and Table 3 show the sex distribution of patients' deformities. They indicate that with the exception of face deformities, male patients presented with more deformities than female patients.

The total numbers and percentages in Tables 2 and 3 and Figures 2 and 3 respectively are expressed relative to the total 603 deformities found in the 159 leprosy patients with deformities. The 603 deformities (410 male, 193 female) is because some of the 159 patients with deformities had one or more deformities.

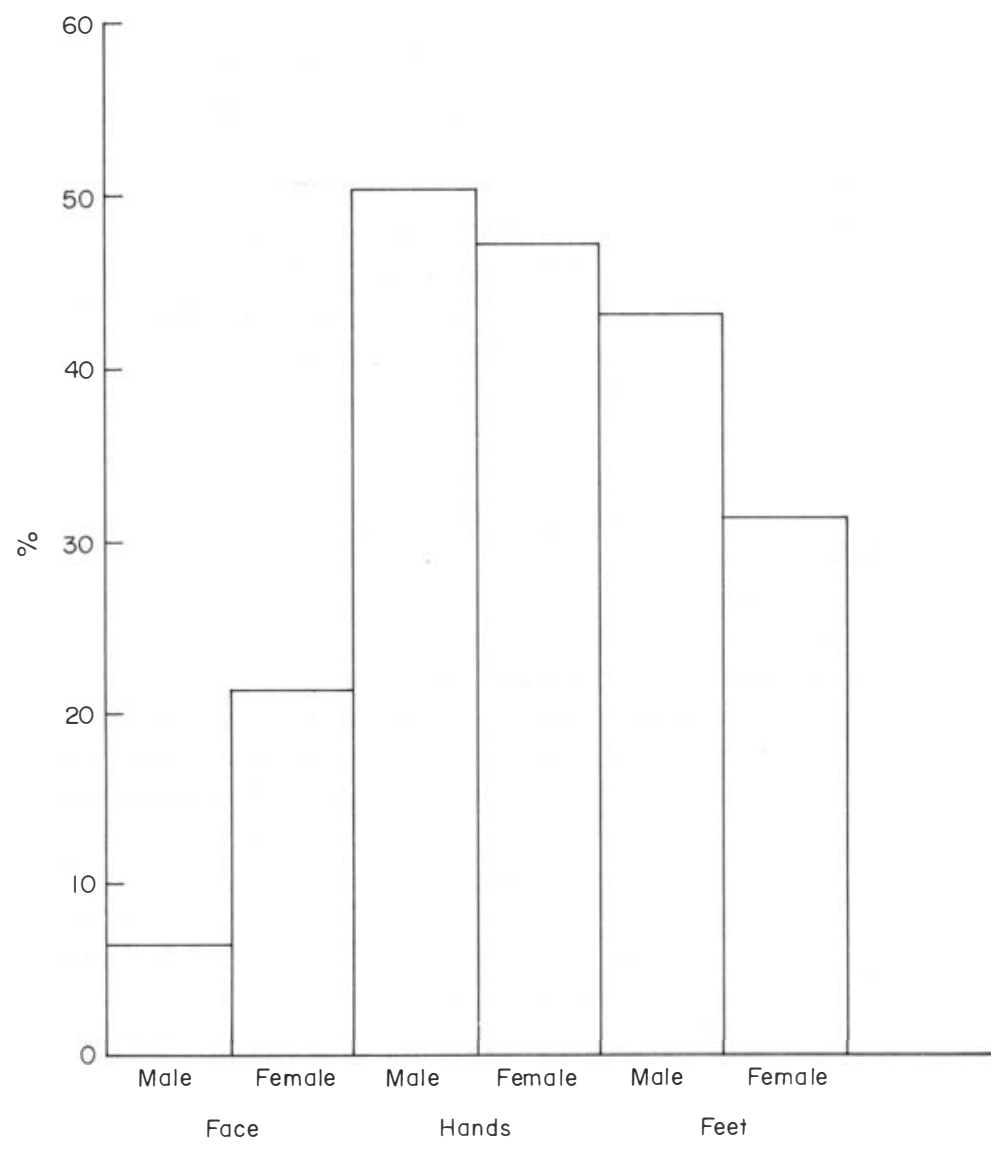

Figure 3. Percentage of male/female distribution of deformities in face, hands and feet amongst 159 leprosy patients with a total of 603 deformities. 


\section{Discussion}

The need to know the prevalence of deformities and an estimate of the number of patients with such deformities in a leprosy population cannot be too strongly emphasized. Such data helps to assess and evaluate the success or failure of the leprosy control programme. The general public and the patients will find it hard to believe that leprosy can be cured if the disability rate remains high.

Deformities in leprosy are of two categories, one is related to the disease process and the other is due to preventable secondary complications related largely to the level of patient care. ${ }^{3}$ Early detection and appropriate management remain the best way to help patients prevent irreversible damage and deformity.

In primary (direct) damage, the nerve may be damaged in the disease process. This can result in sensory loss, muscle paralysis or weakness and dry skin. Sensory loss makes the part of the patient affected very vulnerable to abuse and injuries such as burns and injuries from sharp objects, muscle paralysis could result in stiffness of joints, while dry skin could result in cracked skin and fissures that may eventually develop into ulcers. Burns and other injuries due to anaesthetic parts of the body, stiff joints and ulcers are all secondary complications of the disease. There could be primary damage without these secondary complications. This arrest of nerve and muscle damage at the primary level depends on the adequacy of the patient's management and meticulous self-care on the part of the patient.

This study showed that about $38.78 \%$ of the patients studied had one or more deformities. This figure is not as high as the nearly $50 \%$ reported in another study. ${ }^{9}$ Hand deformities were the most common with $49 \cdot 25 \%$ of all deformities, followed by foot, $39 \cdot 47 \%$ and face, $11 \cdot 28 \%$. This sequence conforms with other studies. ${ }^{10,11}$ Apart from sensory loss of the palms and soles, the three most frequent of all deformities were mobile claw hands, plantar and palmar ulcers.

Sehgal \& Srivastava ${ }^{2,11}$ citing another study ${ }^{12}$ observed that hand deformities accounted for $29 \cdot 1 \%$ of total patients with deformities, also citing further studies ${ }^{13,14}$ claw hands were recorded as $19 \cdot 7 \%$ and $8 \%$ and absorption of fingers $8 \cdot 3 \%$ and $19 \cdot 8 \%$ respectively. The present study however indicates that hand deformities account for $49 \cdot 2 \%$ of all deformities, while claw hand and absorption of fingers account for $12 \cdot 94 \%$ and $3 \cdot 32 \%$ respectively.

The probable reasons for the ulcers could be traced to the nature of farming tools and the footwear of the patients. Most patients are subsistence farmers who use hoes and cutlasses, and trek long distances barefoot or with inappropriate footwear in search of pasture for their animals. Most of them ref use to use hospital handpads when out of sight of hospital workers on the grounds that they prevent an adequate grasp of their farming tools. They do not use protective footwear because they expose the feet of those without toes, and the sandals are well known and associated with leprosy patients.

Water is a major problem in most drought-stricken areas of the state, so foot and general selfcare constitute major problems to the patients.

The challenge now is to develop appropriate handpads for tools, and to design protective footwear that is fashionable and acceptable to patients.

The essence of adequate self-care has to be emphasized to the patients. There is also the need to reach out to patients, persuade and influence them at the earliest stage of the disease to avoid complications, or where they already exist, to avoid their worsening. 
They should not be waited for in the hospitals, they should, where possible, be reached in their communities.

\section{Acknowledgment}

My thanks are due to the Borno State Hospitals Management Board for permission to publish these findings. My profound thanks are also due to Wim $\mathbf{J}$ Brandsma for supplying me with some of the literature, they helped immeasurably in firing my enthusiasm and inspiration. I am no less grateful to sister Paulina M Makum for the stationery. I am also indebted to Peter B Kwajaffa for typing the scripts and the staff of the diagnostic skin clinic for their co-operation.

\section{References}

1 Thangaraj RH, Yawalkar SJ. Leprosy for medical practitioners and paramedical workers. Basel: Ciba-Geigy Ltd, 1986, pp. 1-15, 66-75.

2 Seghal VN, Srivastava G. HD deformities and disabilities: current status. The Star, 1988; 47(5): Part 1, $10-13$.

3 Rao PSS, Karat S, Karat ABA, Furness MA. Prevalence of deformities and disabilities among leprosy patients in an endemic area. Int J Lepr, 1970; 38: 1-11.

4 Brandsma JW, Jong N de, Tjepkema T. Disability grading in leprosy. Suggested modifications to the WHO disability grading form. Lepr Rev, 1986; 57: 361-9.

5 Brunel W, Schecter WP, Schecter G. Hand deformity and sensory loss due to Hansen's disease in American Samao. J Hand Surg, 1988; 13A: 279-83.

6 Wheate HW, Pearson JMH. A practical guide to the diagnosis and treatment of leprosy in the basic health unit. All Africa Leprosy and Rehabilitation Centre, Addis Ababa, Ethiopia, 1979; pp. 18-24.

7 Brand P. Insensitive feet. A practical handbook on foot problems in leprosy. The Leprosy Mission, 50 Portland Place, London WIN 3DG, 1984; pp. 56.

8 Watson JM. Preventing disability in leprosy patients. The Leprosy Mission International, 50 Portland Place, London WIN 3DG, 1986; pp. 25-31.

9 Reddy NBB, Satpathy SK, Krishnan SAR, Srinivasan T. Social aspects of leprosy, a case study in Zaria, Northern Nigeria. Lepr Rev, 1985; 56: 23-5.

${ }_{10}$ Chaturvedi RM. Epidemiological study of leprosy in Malwani suburb of Bombay. Lepr Rev, 1988; 59: 11320.

11 Seghal VN, Srivastava G. HD deformities and disabilities: current status, part 11. The Star, 1988; 47(6): 8-11, 16.

12 Hassan S. A survey of leprous deformities among the patients of Hyderabad city. Lepr India, 1977; 49: 393-8.

13 Noorden SK, Srinivasan H. Deformity in leprosy: An epidemiological study. Ind J Med Res, 1969; 57: 17581.

14 Nagabhushanam P. Gross deformities in leprosy-A group survey. Ind J Derm Ven, 1967; 33: 70-2. 